\title{
EXTRACCIÓN MANUAL DE GARRAPATAS Riphicephalus (Boophilus) microplus EN GANADO BOVINO COMO ESTRATEGIA DE CONTROL ${ }^{1}$
}

\author{
Rodolfo WingChing-Jones ${ }^{2}$
}

\section{RESUMEN}

Se determinó el impacto de la extracción manual de garrapatas sobre la población de individuos por animal durante los años 2009 al 2012, en un hato Jersey dedicado a la producción láctea en Turrialba, Costa Rica. Dos veces por semana, durante el ordeño de la mañana, se contaban y extraían las garrapatas con un tamaño entre 5 y $10 \mathrm{~mm}$ de largo. La información fue registrada en una base de datos de acuerdo al número de identificación del animal, con el día del muestreo, el mes, el año, el aparto de la posible infestación (15 días antes del muestreo), el lado del animal muestreado, la zona anatómica de muestreo y el número de garrapatas recolectadas por área y el total recolectado por día (sumatoria de todas las áreas de muestreo). El conteo de garrapatas por animal fue afectado significativamente por el animal, el mes y el año del muestreo, el aparto de la finca, el número de animales en pastoreo, la zona anatómica de muestreo del animal y el lado del animal $(p<0,05)$. El número de garrapatas por animal durante todo el año fluctuó entre 0,53 y 1,56 garrapatas por animal (por muestreo), siendo mayor en condiciones de mayor precipitación. La técnica redujo en $21 \%$ la población inicial de garrapatas, sin embargo, la implementación de la misma esta condicionada a la cantidad de animales que conforma el hato de la finca y así como a la disponibilidad del personal.

Palabras clave: Ectoparásito, ganado de leche, Jersey, condiciones climáticas

\footnotetext{
${ }^{1}$ Proyecto de investigación 510-A7-805. Módulo Lechero. Empresa Auxiliar 026. Universidad de Costa Rica. San José, Costa Rica.

${ }^{2}$ Universidad de Costa Rica. Escuela de Zootecnia y Módulo Lechero-Sede del Atlántico, Programa de Agricultura Orgánica-UCR. Autor por correspondencia: rodolfo.wingching@ucr.ac.cr 


\section{ABSTRACT}

\section{Manual removal of ticks Rhipicephalus (Boophilus) microplus in cattle as control} strategy. The impact of manual removal of ticks on its population per animal during the years 2006 to 2012, in a Jersey cow dairy production in Turrialba, Costa Rica was determined. During the morning milking, twice a week, ticks with a size between 5 and 10 $\mathrm{mm}$ long were counted and removed. This information was registered in a database according to animal identification number, the sampling day, month, year, the possible infestation paddock (15 days before sampling), animal's sampled side, anatomic sampling zone and number of ticks collected by area and the total collected per day (sum of all sampling areas). The tick count per animal was significantly affected by the animal, month and year of sampling, the paddock of the farm, the number of grazing animals, the area and animal's side $(p<0.05)$. The number of ticks throughout the year fluctuated between 0.53 and 1.56 ticks per animal (per sampling), being higher in conditions of greater rainfall. The technique reduced the initial tick population by $21 \%$; however it is conditioned to the number of animal in the herd as well as personnel availability.

Keywords: ectoparasites, dairy cattle, Jersey, weather conditions

\section{INTRODUCCIÓN}

Las garrapatas en sistemas de producción animal de interés zootécnico son los principales parásitos externos que impactan la productividad de los animales domésticos en condiciones tropicales y subtropicales. Lo anterior, debido a un efecto directo en la succión de sangre, daño al cuero y medio de ingreso de enfermedades producidas por bacterias, virus, rickettsias y protozoarios (Muhammad et al., 2008). Según Álvarez et al. (2003), la garrapatas Riphicephalus (Boophilus) microplus se encuentra en todo Costa Rica hasta una altura no mayor a los $2.000 \mathrm{msnm}$ y hasta una temperatura no menor a los $13^{\circ} \mathrm{C}$. 
En áreas de potrero, las condiciones de baja humedad y alta radiación provocan una disminución en la eclosión de los huevos y supervivencia de las ninfas de las garrapatas antes de llegar a su hospedero (Navas, 2003). También poseen depredadores, los cuales participan como controladores naturales de sus poblaciones, como los nematodos entomopatógenos (Ojeda et al., 2011), las hormigas, hongos, bacterias, roedores, insectos y aves (Barre et al., 1991).

Dentro de las medidas de control que se emplean para combatir este parásito, el uso de acaricidas es la práctica de mayor difusión a nivel de productores y técnicos relacionados con la producción animal, con el agravante de un mal uso de estos, lo cual genera resistencia en las garrapatas, un alto costo del programa sanitario, por un mayor número de aplicaciones, efecto sobre poblaciones no blanco (Álvarez et al., 2008), problemas de contaminación ambiental y presencia de residuos en la leche y en la carne de estos productos (De la Fuente et al., 2007).

Como alternativas a los problemas de residualidad y de resistencia que se presentan con los productos sintéticos (acaricidas) para el manejo de las garrapatas, se desarrollaron estrategias como el uso de vacunas (De la Fuente et al., 2007), pastoreo en conjunto con aves domésticas (Dreyer et al., 1997), conteo de individuos para aplicar baños con desparasitantes (De la Vega et al., 2009), uso de hierbas con acción insecticida y acaricida (Chungsamarnyart y Jansawan, 2001), manejo de pasturas (pastoreo rotacional o quemas) (Gómez et al., 2008), extracción manual, animales transgénicos con genes de resistencia (Gosh et al., 2007), uso de nuevas generaciones de acaricidas, uso de razas bovinas resistentes y el uso de hongos entomopatógenos (Leemon et al., 2008).

En Costa Rica se evaluó el impacto que presentan extractos vegetales sobre la mortalidad de teleóginas de $B$. microplus; la reducción de la oviposición (reducción de la relación huevos/garrapatas) y la disminución de la eclosión (Álvarez et al., 2008), como un control alternativo de garrapatas.

En relación al uso de hongos entomopátogenos, se evaluó el impacto del hongo Metarhizium sp. en condiciones in vivo e in vitro (Arguedas et al., 2008 y Rojas et al., 2008) sobre la vitalidad de adultos. Po otro lado, un único trabajo descrito por Álvarez y Rojas (2011), describe el impacto de la extracción manual de garrapatas sobre la 
población de estos parásitos en la carga parasitaria de animales Holstein y Jersey en Santa Clara de San Carlos, Costa Rica.

Por tal motivo, el objetivo de este trabajo fue determinar el impacto de la extracción manual de individuos sobre la población de garrapatas del género Riphicephalus (Boophilus) microplus en animales Bos taurus de la raza Jersey en condiciones de trópico húmedo.

\section{MATERIALES Y METODOS}

\section{Ubicación del sistema productivo}

El Módulo Lechero de la Sede del Atlántico de la Universidad de Costa Rica, se ubica en Turrialba, cantón número 5 de la provincia de Cartago, a una altura cercana a los 700 msnm. Es un sistema de producción láctea, que cuenta con 42 animales Jersey, de los cuales en promedio, ordeña 16 animales por año, dos veces al día.

Las vacas pastorean 25 apartos de pasto Estrella Africana, con una dimensión promedio de 1.000 metros cuadrados, lo que corresponde a una disponibilidad de 62,5 $\mathrm{m}^{2} /$ animal/día. Además del aporte de forraje, se suplementa por día cada animal con cinco kilogramos de alimento balanceado (16 \% PC y $3.000 \mathrm{kcal}$ ED/kg), $100 \mathrm{~g}$ de grasa sobrepasante (Jabones cálcicos), $100 \mathrm{~g}$ de sal blanca y $100 \mathrm{~g}$ de premezcla mineral.

\section{Descripción de la extracción: garrapatas, momento y secuencia}

El tamaño mínimo por garrapata para realizar la extracción fue 5 - 10 mm de largo, lo cual permite la extracción de individuos adultos repletos o en proceso de repleción (Figura 1) de forma manual.

Para conocer la población de individuos bajo un sistema químico de control de ectoparásitos, durante tres años se realizaron conteos de garrapatas cada 30 días en los animales antes de la realización de los baños con acaricidas comerciales, actividad que inicio en el año 2006. A inicios del año 2009, se elimina la práctica de los baños contra garrapatas en el sistema de producción y es sustituida por la extracción de los individuos 
de forma manual, actividad que se realizó durante el ordeño de la mañana, los días martes y viernes, hasta finales del mes de Julio del 2013, lo que genera 4,5 años de investigación. Para facilitar la extracción, esta se realizó en el lado derecho del animal el cuál es el área expuesta a los ordeñadores.

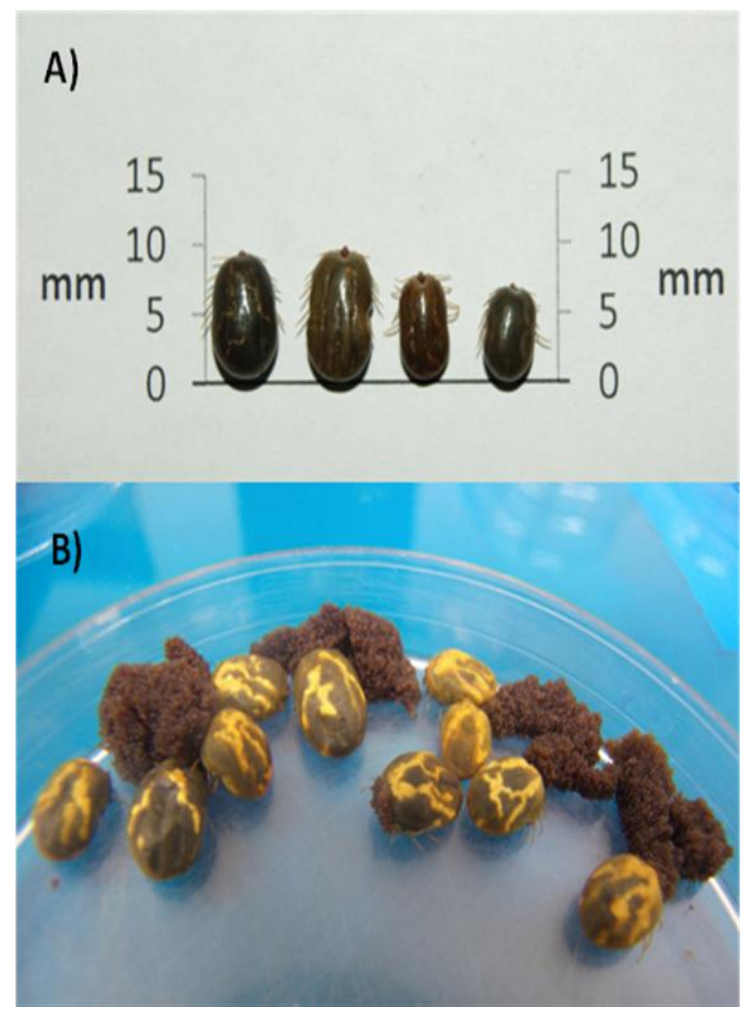

Figura 1. A) Tamaño y características de las garrapatas extraídas de forma manual según los criterios de investigación y, B) magnitud de la ovoposición de una garrapata adulta.

\section{Condiciones ambientales imperantes durante el periodo de investigación}

En cuanto a condiciones ambientales, se recolectaron datos de precipitación ( $\mathrm{mm}$ ), humedad relativa $(\%)$, temperatura $\left({ }^{\circ} \mathrm{C}\right)$ y radiación $\left(\mathrm{MJ} / \mathrm{m}^{2}\right)$. La información fue facilitada por el Centro Agronómico Tropical de Enseñanza e Investigación (CATIE), en donde se describen las condiciones promedio para el cantón de Turrialba, desde el año 1980 (Figura 2). Para los años que comprenden el periodo de investigación (2006-2013), se realizó un análisis de regresión, por medio del programa PROC GLM (SAS, 2003), para 
determinar los efectos que presenta la variación entre años para estas variables climáticas. Cuando se determinaron efectos significativos, la separación entre medias se realizó mediante la prueba de Duncan, con una probabilidad p<0,05 (SAS, 2003).

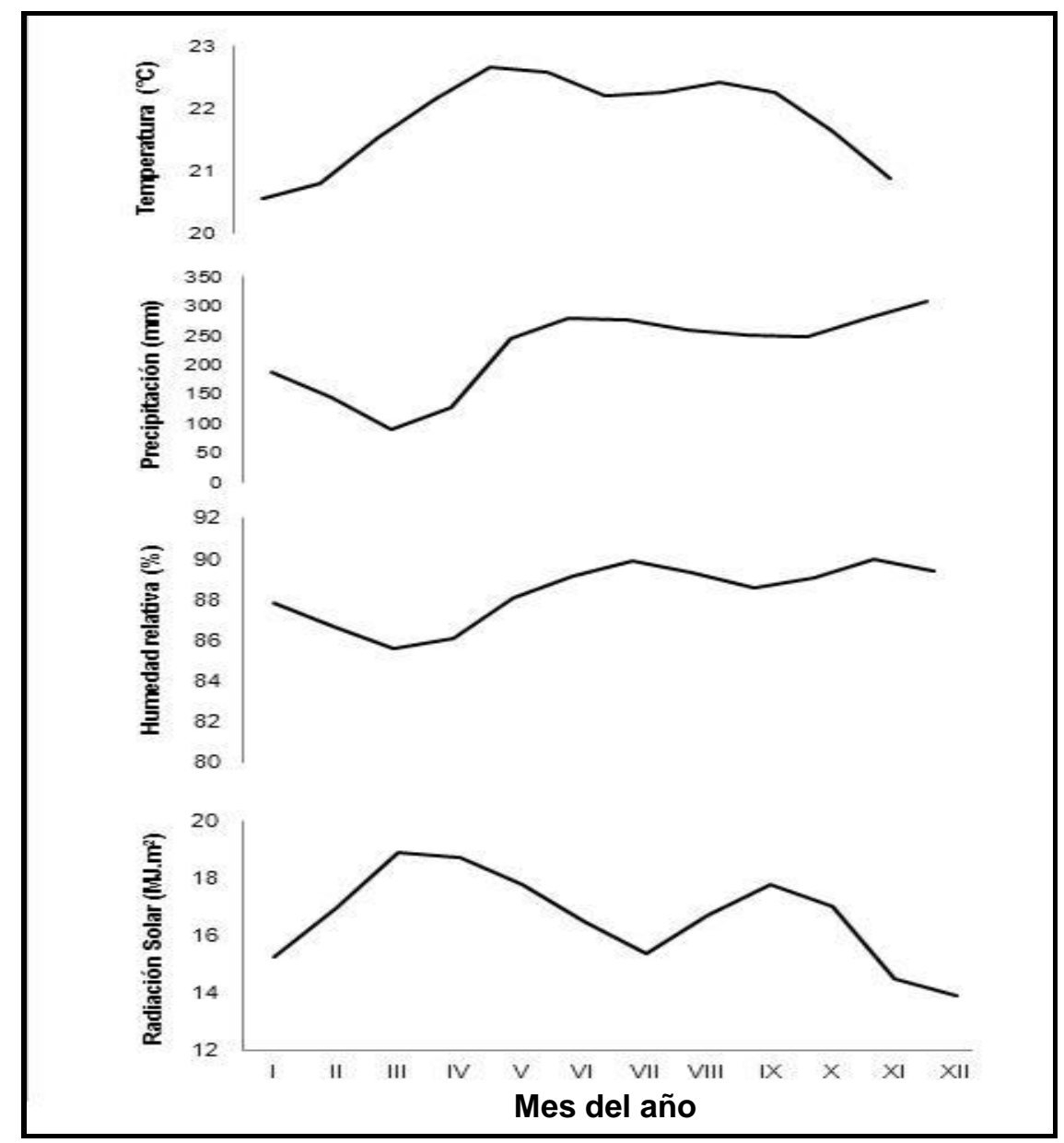

Figura 2. Promedios de la variación durante el año de la precipitación, humedad relativa, radiación y temperatura desde 1980 hasta 2013 en Turrialba, Cartago, Costa Rica.

\section{Organización y análisis de la información recolectada}

Los datos recolectados se ordenaron con ayuda de una hoja de Microsoft Excel $₫(2010)$, en donde, para cada columna se digitó la información según el número de identificación del animal, el día del muestreo, el mes, el año, el aparto posible de infestación (15 días antes del muestreo), el lado muestreado, la zona anatómica de muestreo y el número de 
individuos recolectados por zona y el total recolectado por día (sumatoria de todas las áreas de muestreo).

En el caso de las zonas anatómicas de muestreo del animal se consideró: la parte derecha de la cabeza, la oreja, el pescuezo, la axila, la zona ventral, la ubre, el rabo, la nalga y la vulva. Mientras que, en el caso del lado, este se dividió en derecho e izquierdo. En el caso de los conteos en el lado izquierdo, no se separaron según la zona anatómica de muestreo, solo se realizó un conteo total de garrapatas. Mediante el programa Proc GLM de SAS (2003) se determinó el impacto que presentan las variables independientes (número de identificación del animal, el día del muestreo, el mes, el año, el aparto, el lado muestreado, la zona anatómica de muestreo) sobre el número de individuos contabilizados en cada zona anatómica de muestreo, así como en el conteo total. Cuando se determinaron diferencias entre medias, se procedió a aplicar la prueba de Duncan, la cual permite obtener diferencias con una probabilidad de $p<0,05$.

\section{RESULTADOS Y DISCUSIÓN}

\section{Condiciones climáticas predominantes durante los años de estudio}

Se presentaron periodos de condiciones climáticas propias al fenómeno del Niño y de la Niña, los cuales podrían influir en la dinámica del ciclo de vida de las garrapatas, el ciclo de sus depredadores naturales, así como el comportamiento de las vacas. Según los resultados obtenidos, solo la radiación y la temperatura, presentan variación $(p<0,05)$ según el año evaluado (Cuadro 1). No obstante, la precipitación no varió significativamente, esta variable podría presentar un impacto sobre la dinámica de las garrapatas, la cual se relaciona con el crecimiento de las pasturas y las condiciones de humedad en la superficie del suelo, lugar donde se realiza la ovoposición. Por tal motivo, no se considera el impacto de las condiciones climáticas según el año en la discusión de este trabajo, y se hace mención al comportamiento de la precipitación descrita en la Figura 2, la cual presenta una tendencia a incrementar en el segundo semestre de cada año. 
Cuadro 1. Promedios de precipitación, humedad relativa, temperatura y radiación en Turrialba, Cartago, durante los años 2006 - 2013.

\begin{tabular}{ccccc}
\hline Año & $\begin{array}{c}\text { Precipitación } \\
\left(\mathrm{mm} / \mathrm{mes}^{\mathrm{n}}\right)\end{array}$ & $\begin{array}{c}\text { Humedad } \\
\text { relativa* }(\%)\end{array}$ & $\begin{array}{c}\text { Temperatura } \\
\left({ }^{\circ} \mathrm{C}\right)\end{array}$ & $\begin{array}{c}\text { Radiación } \\
\left(\mathrm{MJ} / \mathrm{m}^{2}\right)\end{array}$ \\
\hline 2006 & $230,01^{\mathrm{a}}$ & $87,37^{\mathrm{a}}$ & 22,04 & $16,49^{\mathrm{ab}}$ \\
2007 & $223,3^{\mathrm{a}}$ & $89,07^{\mathrm{c}}$ & 22,23 & $16,15^{\mathrm{ab}}$ \\
2008 & $252,38^{\mathrm{a}}$ & $90,25^{\mathrm{c}}$ & 21,99 & $15,27^{\mathrm{b}}$ \\
2009 & $236,2^{\mathrm{a}}$ & $91,97^{\mathrm{b}}$ & 21,91 & $16,50^{\mathrm{ab}}$ \\
2010 & $272,85^{\mathrm{a}}$ & $93,07^{\mathrm{ab}}$ & 22,20 & $15,38^{\mathrm{ab}}$ \\
2011 & $228,34^{\mathrm{a}}$ & $93,02^{\mathrm{ab}}$ & 21,98 & $16,92^{\mathrm{a}}$ \\
2012 & $219,89^{\mathrm{a}}$ & $93,71^{\mathrm{a}}$ & 21,89 & $16,16^{\mathrm{ab}}$ \\
2013 & $162,08^{\mathrm{b}}$ & $93,67^{\mathrm{a}}$ & 22,32 & $17,02^{\mathrm{a}}$ \\
\hline
\end{tabular}

*Letras diferentes en la misma columna, indican diferencias estadísticas $p<0,05$

\section{Dinámica de población de garrapatas repletas de huevos durante el periodo experimental}

El conteo de individuos fue afectado significativamente según el animal muestreado, el mes y el año del muestreo, el aparto de la finca, el número de animales en pastoreo, zona anatómica de muestreo del animal y el lado del animal $(p<0,05)$.

En el caso del efecto por animal, de los 50 animales muestreados en el periodo de la investigación, un único animal presentó un promedio de 45 garrapatas (por muestreo), valor que supera en 20 veces al promedio de la población de garrapatas obtenidas por animal. Este comportamiento indica la susceptibilidad al parasitismo de este animal y esta se ratifica al revisar los registros sanitarios de la finca. La madre de esta vaca presentó una afinidad similar para ser hospedera, asociada a una reincidencia a padecer enfermedades como la anaplasmosis. En contraste con este único caso, se registró la presencia de animales con conteos menores a 0,5 garrapatas por animal (por muestreo), variables que permiten hacer una selección de los animales por su resistencia al presentar conteos altos de estos parásitos. 
Según el mes de muestreo, se observa en la Figura 3 una tendencia de crecimiento de la población de garrapatas por animal a partir del mes de junio, debido a una mayor productividad de la pastura, que se podría relacionar a una mejor distribución de la lluvias y una menor radiación solar en la zona de estudio, condiciones que permiten un mejor desarrollo del ciclo de vida de las garrapatas (Navas et al., 2003). Esta misma conclusión podría explicar el número de garrapatas repletas observadas en el mes de mayo, donde al iniciar las lluvias (Figura 2), se generan condiciones que propician el desarrollo de las garrapatas y sus depredadores naturales en los meses siguientes.

También se denota un descenso de la población de garrapatas entre los meses de diciembre y enero. Lo anterior podría estar relacionado a un problema de alta precipitación en la comunidad de Turrialba en el periodo de diciembre. Esto provoca un mayor pisoteo de la pastura, debido a que se observan encharcamiento del agua y generación de puntos de barro o lodo, condiciones adversas para el desarrollo y eclosión de huevos que se ovopositan durante estos periodos (Figura 2 y 3). Ligado a esta situación, se observa un periodo de transición entre alta a baja precipitación en el mes de enero, lo cuál reduce el crecimiento de los forrajes, aumenta la radiación en los potreros y disminuye la humedad en los suelos, variables adversas para la sobrevivencia de los huevos de garrapatas (Navas, 2003).

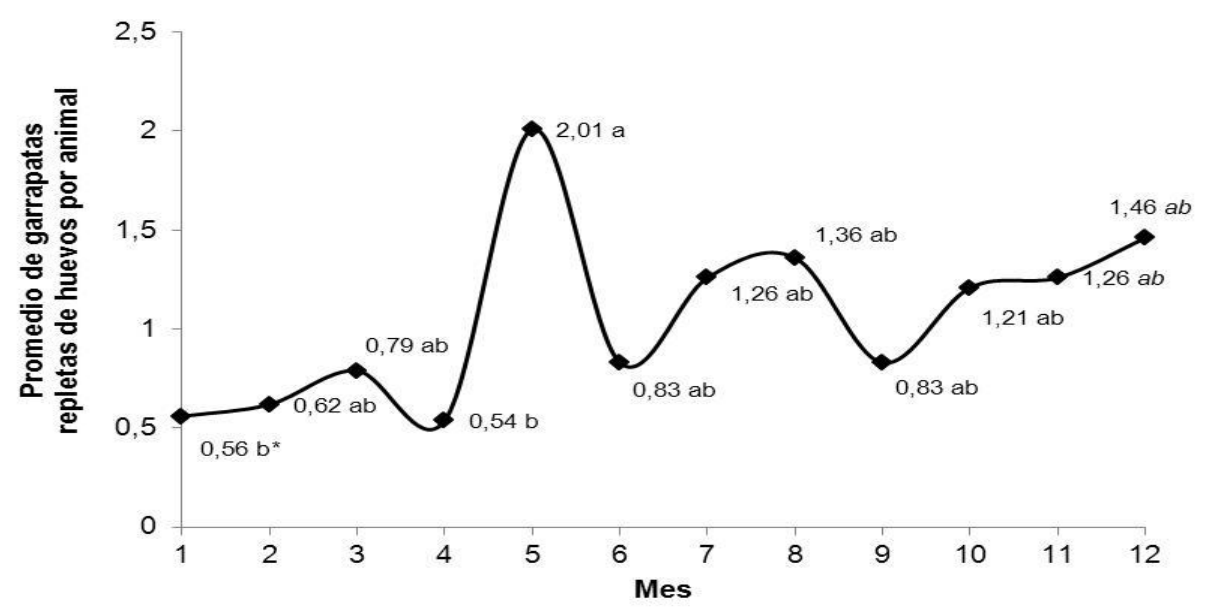

*Letras diferentes indican diferencias entre medias según la prueba de Duncan $(p<0,05)$

Figura 3. Promedios por muestreo de la dinámica de población de las garrapatas repletas de huevos en bovinos según el mes de estudio. Turrialba, Cartago, Costa Rica, 2009 - 2013. 
Con respecto a la zona de potreros o pastoreo, se obtienen valores máximos y mínimos entre 3,60 y 0,34 garrapatas por animal (por muestreo) respectivamente, lo cual indica que la población de garrapatas esta distribuida de diferente forma en el sistema de producción, situación que permite desarrollar un plan de manejo de los animales, según el área de la finca en que se encuentre. No se encontró ninguna relación con la cantidad de garrapatas por animal y las características del potrero, como la densidad de la cerca viva y la sombra que esta genera.

Por otro lado, con el número de animales pastoreando por día, se obtuvo una tendencia cuadrática, la cual presentó el mayor número de garrapatas por animal entre 14 y 16 animales en pastoreo. Por lo que se podría suponer, que por encima de 16 animales en pastoreo, se da un efecto negativo sobre la eclosión de los huevos por pisoteo de los animales, mientras que, por debajo de 14 animales, se generan espacios en el aparto, donde los animales no pasan, y se reduce la posibilidad que estos ácaros puedan subirse al semoviente.

Por ultimo, según el lado del animal evaluado, se determinó una población de 1,56 y 0,55 garrapatas por animal (por muestreo) para el lado derecho e izquierdo, respectivamente. En cambio, se determinó una mayor cantidad de individuos según el área del animal muestreada, en donde la ubre presentó una mayor cantidad de individuos en promedio que las otras partes (0,33 individuos por animal/por muestreo), seguida del pescuezo y la nalga (0,20 garrapatas por animal/por muestreo), en tercer lugar de afinidad por las garrapatas se definió el área de la axila (0,12 garrapatas por animal/por muestreo), en cambio la zona ventral, la vulva y la base del rabo se agruparon en cuarto lugar (0,6 garrapatas por animal/por muestreo).

Adicionalmente, una menor población de garrapatas por individuos se describe en la oreja y la cabeza (0,05 garrapatas por animal/por muestreo). Esta información difiere a la presentada por De la Vega et al. (2009) quienes informan no encontrar diferencias entre las áreas del animal muestreada a razón del número de individuos contados, comportamiento que asocian a las características del hospedero y a condiciones ambientales. En esta investigación, los resultados podrían estar explicados por una mayor protección del parásito en el caso de la ubre (menor radiación directa y mayor humedad) y caso contrario el caso de la oreja y la cabeza, puntos de mayor exposición. 


\section{Impacto de la extracción manual de garrapatas repletas sobre el conteo en los animales}

En los tres primeros años de control químico de la población de garrapatas por animal, se observa una variabilidad significativa entre poblaciones para los años 2006, 2007 y 2008 (Figura 4). Lo cual se podría relacionar a la dinámica poblacional que presentan las garrapatas de forma natural en un sistema de control con productos acaricidas y la rotación de ingredientes activos. A partir del año 2009, se observa el impacto de la extracción manual de las garrapatas de forma significativa $(p<0,05)$ y del reservorio de garrapatas que presenta la finca en los apartos, debido a que esta técnica se aplicó durante 10 meses en ese año y la población de garrapatas, siempre fue mayor a un individuo por animal por muestreo.

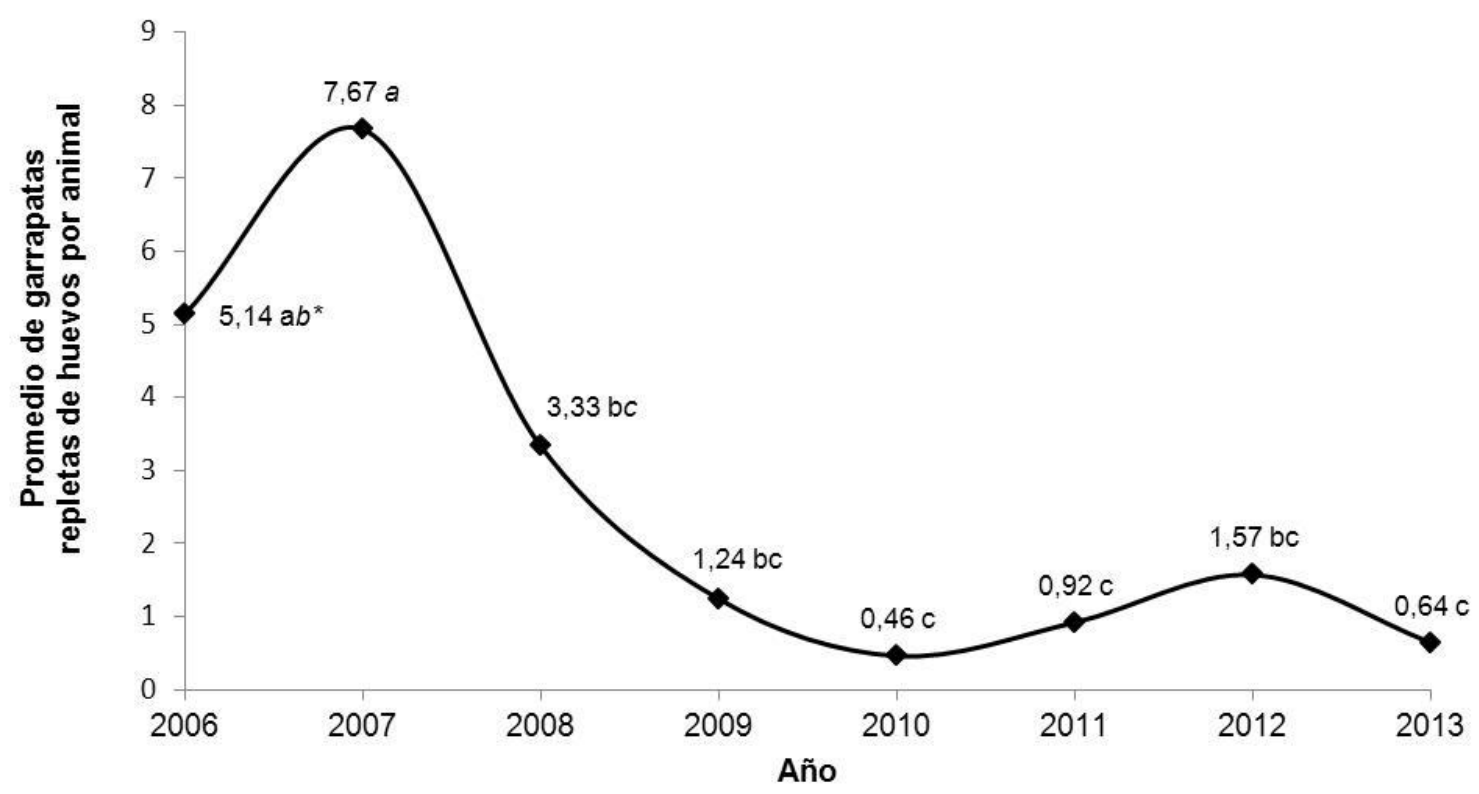

*Letras diferentes indican diferencias entre medias según la prueba de Duncan $(p<0,05)$

Figura 4. Número de garrapatas repletas de huevos extraídas en promedio (por muestreo) al mes por animal según el año. Turrialba, Cartago, Costa Rica, 2006 - 2013.

Por el contrario, para los años 2010, 2011, 2012 y 2013 se describe una tendencia a mantener una población menor o igual a una garrapata por animal, donde la técnica permite a este sistema de producción ser libre del uso de productos sintéticos para el 
control de garrapatas. El efecto directo de esta técnica, se da sobre la ovoposición de los huevos de garrapatas en el potrero por cada garrapata (Figura 1), lo cual permite generar una reducción del 100\% de la eclosión; o el equivalente a 20.000 huevos por cada gramo de postura que se dejan de poner en los potreros, al eliminar las hembras repletas antes de que estas se suelten del hospedero para culminar su ciclo de vida (Arguedas et al., 2008). Así, esta técnica permite implementar un manejo integrado de plagas en finca, debido a que se impacta la estrategia reproductiva de las garrapatas, la cuál es, una abundante progenie en poco tiempo (Horn, 1988).

Si bien se determina el impacto beneficioso de esta técnica sobre la población de individuos en el animal y el reservorio de estos parásitos en los apartos, esta alternativa de control podría estar limitada por el tamaño del hato o la cantidad de personal presente en el sistema de producción, en esta investigación el tiempo que se empleó en el conteo y extracción de las garrapatas en cada animal fue de dos minutos, valor que concuerda con lo informado por Álvarez y Rojas (2011). Por tal motivo, para implementar esta alternativa de control es importante conocer el sistema de producción a nivel de disponibilidad y motivación del personal y del manejo de los animales. Así reducir el ingreso de insumos al sistema, disminuir la posibilidad de intoxicación del personal, la contaminación ambiental por deriva de producto y mantener o mejorar la inocuidad de la leche o carne producida.

\section{AGRADECIMIENTOS}

Se agradece al Dr. Victor Álvarez y al M.Sc. Augusto Rojas por los aportes y recomendaciones brindadas durante la ejecución y escritura de este trabajo. A Roberto Ugalde y Oscar Garita funcionarios del Módulo Lechero en el apoyo en los periodos de conteo y extracción de garrapatas durante el ordeño de los animales. 


\section{LITERATURA CITADA}

Álvarez, V., Rojas, N. 2011. Recolección manual de garrapatas. Método alterno de combate de esta plaga del ganado. Boletín Parasitología 12(4): 3-4.

Álvarez, V., Bonilla, R., Chacón, I. 2003. Frecuencia relativa de Boophilus microplus (Acari: Ixodidae) en bovinos (Bos taurus y $B$. indicus) en ocho zonas ecológicas de Costa Rica. Revista Biología Tropical 51(2):427-434.

Álvarez, V., Loaiza, J., Bonilla, R., Barrios, M. 2008. Control in vitro de garrapatas (Boophilus microplus; Acari: Ixodidae) mediante extractos vegetales. Revista Biología Tropical 56(1): 291-302.

Arguedas, M., Álvarez, V., Bonilla, R. 2008. Eficacia del hongo entomopatógeno Metarhizium anisopliae en el control de Boophilus microplus (Acari: Ixodidae). Agronomía Costarricense 32(2): 137-147.

Barre, N., Mauléon, H., Garris, G., Kermarrec, A. 1991. Predators of the tick Amblyomma variegatum (Acari: Ixodidae) in Guadeloupe, Frech West Indies. Experimental \& Applied Acarology 12: 163-170

Chungsamarnyart, N., Jansawan, W. 2001. Effect of Tamarindus indicus L. against the Boophilus microplus. Kasetsart. Journal of Natural Science 35: 34-39.

De la Fuente, J., Almuzán, C., Canales, M., Pérez, J., Kocan, K., Willadsen, P. 2007. A ten-year review of comercial vaccine performance for control of control of tick infestations on cattle. Animal Health Research Reviews 8(1): 23-28.

De la Vega, R., Díaz, G., Camejo, A., García, I. 2009. Application of tick sampling on acaricidal bath frecuency in Rhipicephalus (Boophilus) microplus (Acari: Ixodidade). Revista Salud Animal 31(2): 105-107.

Dreyer, K., Fourie, L., Kok, D. 1997. Predation of livestock tics by chickens as a tick control method in a resource poor urban environment. Onderstepoort Journal Veterinarian Research 64(4): 273-276.

Gómez, M., Moguel, M., Rodríguez, C. 2008. Alternativas al manejo de la garrapata Boophilus microplus en el trópico. Universidad Autónoma de Chapingo, CIIDRI y Red mexicana de Tianguis y Mercados orgánicos. p 151.

Gosh, S., Azhahianambi, P., Yadav, M. 2007. Upcoming and future strategies of tick control: a review. Journal of vector Borne Diseases. 44:79-89. 
Horn, D. 1988. Ecological approach to pest management. The Guilford Press. NY, USA. p 285.

Leemon, D., Turner, L., Jonsson, N. 2008. Pen studies on the control of cattle tick (Rhipicephalus (Boophilus) microplus) with Metarhizium anisopliae (Sorokin). Veterinary Parasitology 156:248-260

Muhammad, G., Naureen, A., Firyal, S., Saqib, M. 2008. Tick control strategies in dairy productions medicine. Pakistan Veterinary Journal. 28(1):43-50

Navas, A. 2003. Influencia de la cobertura arbórea de sistemas silvopastoriles en la distribución de garrapatas en fincas ganaderas en el bosque seco tropical. Tesis de maestría. CATIE, Cartago, Costa Rica, 79 p.

Ojeda, M., Rodriguez, R., Galinda, E., Lezama, R., Cruz, C. 2011. Control de Rhipicephalus microplus (Acari: Ixodidae) mediante el uso del hongo entomopatógeno Metarhizium anisopliae (Hypocreales: Clavicipitaceae). Revisión. Revista Mexicana de Ciencias Pecuarias 2 (2):177-192

Rojas, A., WingChing, R., Acuña, O. 2008. Prueba de eficacia de hongos entomopatógenos en el control de garrapatas Boophilus microplus. Informe Final. Proyecto 739-A5-043. Vicerrectoría de Investigación. Universidad de Costa Rica. Costa Rica. p 14.

SAS. 2003. SAS 9.1.3 for Windows. Service Pack 4.Win_Pro plataforma. Copyright (C) 2002-2003 by SAS Institute Inc. Cary, N.C. USA. 